\title{
A Survey on Isolated Word and Digit Recognition using Different Techniques
}

\author{
Pooja Prajapati \\ PG Student \\ Information Technology Department, \\ G H Patel Collage of Engineering and Technology, \\ India
}

\begin{abstract}
Nowadays, Spoken digit recognition is one the challenging task in the field of speech recognition. Spoken digit recognition is necessary nowadays in many applications that needed number as input like telephone dialing using speech, addresses, airline reservation $\&$ automatic directory to retrieve $\&$ send information which make the system more efficient to use. Also, It proves very helpful for physically challenged people in hands \& eyes free applications. Various techniques are used for isolated speech recognition like MFCC, HMM, LPC. But among all of them many researchers found that MFCC is widely used \& give a more accurate result. ASR achieves a maturity level in many Indian languages. Mostly research work has been carried out. Here in this paper, Discussions of the survey is on some of that recent research work in isolated digit recognition for the Indian languages like English, Gujarati, and Hindi \& also in other similar languages. Likewise, discussing different approaches, methods \& comparative analysis about recent research work done in isolated digit \& word recognition in various languages.
\end{abstract}

\section{General Terms}

Speech Recognition, Isolated digit recognition

\section{Keywords}

Speech Recognition, MFCC, Hidden Markov Model (HMM), LPC, Isolated word, isolated digit recognition.

\section{INTRODUCTION}

Speech recognition is a very common \& useful way to interact with others. In today's modern world, many devices are designed \& produced user friendly for the communication purpose, speaking activity $\&$ also used for many applications which make devices very reliable \& more convenient to use. Speech recognition is the interface between human \& machine interaction. It is a natural language which is based on human voice which help machine to recognize \& understand various human languages spoken by different speakers by means of Speech recognition. It can be done by converting speech signals into a sequence of words by means of an algorithm. Speech recognition is also known as Automatic Speech Recognition (ASR) system [42], [44]. The Aim of the speech recognition area is to develop system for various languages by using various techniques by converting speech into machine readable format. Which provide more convenient \& handsfree environment for users.

Speech recognition, increase technology \& encourages people who are not physically \& mentally comfortable to operate machines due to the lack of the knowledge \& language barrier also for disabled \& blind people [46]. It is very challenging task for blind people to read from the machine. Also, for the foreign students because of the pronunciations of different

\author{
Miral Patel \\ Professor \\ Information Technology Department, \\ G H Patel Collage of Engineering and Technology, \\ India
}

speakers \& grammar rules. Various issues like Noisy environment, Transducer, a different speech style which affects the speech [42], [43]. A speech recognition system developed to overcome these types of difficulties during recognition.

This paper organized in following manner, Section 1 , is about Introduction part. Section 2 presents the scope \& motivation of this survey paper, Section 3 is a brief definition about the speech which is also known as "Classification of Speech Recognition", Section 4 is Literature Survey about recent contribution made in isolated word \& digit recognition system in different languages, Section 5 focuses on techniques used in isolated speech recognition, Section 6 is analysis \& discussion, Section 7 is about Conclusion and future work.

\section{SCOPE AND MOTIVATION}

All This survey paper gives an overview about isolated speech recognition systems developed in different languages, using different techniques with their recent development. It also included an overview about mainly used techniques in isolated speech recognition, their experimental results \& the Graphical representation about the overall work done in the isolated digit recognition system at various languages. Which may help a lot to the researchers to get the overview about the isolated digit recognition system developed in different languages around the world \& the recognition rate achieved by that system helps researchers in choosing the technique that gives better improvements in the isolated digit recognition system.

From the comparative analysis part of this survey paper, it is observed that the isolated digit recognition system is implemented in English language. Little work is done in other similar languages like Gujarati, Hindi, Bengali, Tamil etc. So we conclude with the decision for isolated digit recognition, apart from those languages, requires more attention.

\section{CLASSIFICATION OF SPEECH RECOGNITION}

There is a large amount of variety in speech recognition. This is important to understand the differences between various types of speech utterances, the size of the vocabulary, speaker dependency. Also, its applications \& issues.

\subsection{Types Of Speech Recognition}

\subsubsection{Isolated Word}

It accepts single words or single utterances at a time.

\subsubsection{Connected Word}

Run together minimum pause between them. 


\subsubsection{Continuous speech}

Continuous speech recognizers allows user to speak naturally, while the computer determine the continue Recognizer with continues speech capabilities.

\subsubsection{Spontaneous speech}

System with spontaneous speech ability should be able to handle a variety of natural speech feature such as words being run together.

\subsection{Vocabulary Size}

Vocabulary size of ASR system will affect the system complexity \& also the accuracy of the system because of the limitations of the size at some applications. Types of vocabulary size can be classified as,

Table 1. Vocabulary Size

\begin{tabular}{|l|l|}
\hline Type of vocabulary & No of words contain \\
\hline A Small vocabulary & 10 \\
\hline Medium vocabulary & 100 \\
\hline Large vocabulary & 1000 \\
\hline Very large vocabulary & 10,000 \\
\hline
\end{tabular}

\subsection{Types Of Speaker Model}

\subsubsection{Speaker dependent}

Speaker dependent system mainly developed for a single speaker. This is easy to develop, cheaper \& more accurate. It uses a unique feature of a single person's voice. An upcoming user must have to train system first, by speaking with it so that the system will analyze that how a person talks. This is not as flexible as speaker independent \& speaker adaptive.

\subsubsection{Speaker independent}

In speaker independent, System developed for any speaker of a particular type. It will recognize speech patterns of a large group of people. This system is difficult to develop, expensive $\&$ gives less accuracy than speaker dependent system.

\subsubsection{Speaker adaptive}

A speaker adaptive system developed to adapt its operation to the characteristics of a new speaker. This system is flexible.

\subsection{Speech Recognition Issues}

There are various issues in speech recognition, which are,

\subsubsection{Environment}

Due to the type of noise, signal/noise ratio, working condition.

\subsubsection{Transducer}

Due to microphone

\subsubsection{Speaker}

Speaker independent/dependent, sex, age

\subsubsection{Channel}

Due to the band amplitude, distortion

\subsubsection{Speech Style}

Due to voice tone (quite, normal, shouted)

\subsubsection{Vocabulary}

Due to the characteristics of available data, vocabulary [42], [43], [44], [45], [46], [11], [8], [18]

\subsection{Speech Recognition Applications}

\subsubsection{Educational purpose}

For teaching students of foreign languages to pronounce vocabulary correctly

\subsubsection{Medical sector}

For health care

\subsubsection{Military sector}

For high performance fighter aircraft, battle management.

\subsubsection{Telephony and other domains}

For dial up connection

\subsubsection{Serving the disabled}

\subsubsection{Home automation}

\subsubsection{Automobile audio systems [42], [44], [ 46]}

\section{LITERATURE SURVEY}

This section of the paper highlights literature survey on recent research work has been done in isolated digit recognition in different languages as well as for isolated word recognition. Here some research \& development related to isolated digit \& word are discussed,

Choudhary, Chauhan, \& Gupta, (2013) have implemented automatic speech recognition system for isolated \& connected words of Hindi language using hidden Markova model toolkit (HTK), that is based on Hidden Markov Model (HMM), which is a statistical approach is used to develop the system for 100 distinct Hindi words (small vocabulary). MFCCC is used here for feature extraction which shows an overall system accuracy rate for isolated word is $95 \%$ \& for connected words it is $90 \%$ [1].

Singhal \& Dubey, (2015) have implemented automatic speech recognition for connected words using DTW/HMM for English /Hindi languages. In which author shows their work on isolated word recognizer for speaker dependent data works in both English as well as Hindi using DTW \& HMM techniques, Both techniques are compared in this paper, including database for 10 speaker about 20 isolated word for both languages. which shows Overall WER (Word error rate) for English language is $13.33 \%$ using DTW \& $6.67 \%$ using HMM \& Overall WER for Hindi language is $13.33 \%$ using DTW \& $13.33 \%$ using HMM. From the results author concluded here that HMM model for English language gives most efficient results compared with the DTW approach with minimum WER [2].

H B Chuahan, Prof B A tanawala (2015) have compared both MFCC \& LPC method under vector quantization(VQ) method at comparative study of MFCC \& LPC Algorithms for Gujarati isolated word recognition in which they use database of both male \& female voices where each word is repeated at 5 times by speakers where results shows that using LPC, accuracy is above $85 \%$, MFCC is more above $95 \%$. So author concluded here that MFCC performs better for feature extractor [3].

Pandit \& Bhatt,(2014) have described automatic speech recognition of Gujarati digits using dynamic time warping , In which author discussed speech recognition of Gujarati isolated digit with the database of small vocabulary about 1 to 10 Gujarati digits from 10 speakers. Where MFCC is used for feature extraction \& recognition of unknown speech signal is go through DTW method. Whole this process is done using 
MATLAB. From Experimental results author got the accuracy about $84.44 \%$, while with extra care during recoding they achieve an accuracy rate increased to $95.56 \%$ [4].

Chapaneri \& Jayaswal, (2013) has present efficient speech recognition system for isolated digit in which they discussed speaker independent 0 to 9 English digits in which implementation is done with the IFDTW(improved feature for DTW) method with 13 Weighted MFCC coefficients for feature extraction. Also, they proposed sola based technique for reducing the complexity of recognition. Author founds sola based technique \& faster implementation of IFDTW is 22 times faster than the other techniques which got $99.16 \%$ accuracy [5].

Patel \& Desai, (2014) have described recognition of spoken Gujarati numerals \& its conversion into electronic form, In that proposed model includes mainly three components which are, digitization , feature extraction \& pattern classification. Also, they used MFCC for feature extraction it includes framing, windowing, fast Fourier transform (FFT) \& also compute the Discrete Cosine Transform (DCT) to produce feature vector of spoken numeral. Model work only for Gujarati numeral which is an isolated word that is recognize by different speakers. The proposed model achieves average an accuracy rate of $78.13 \%$ [6].

Elouahabi, Atounti, \& Bellouki, (2016) have described amazigh isolated word speech recognition system using hidden markov model toolkit (HTK), where amazigh language is known as Berber or Tamazight that is so vast in Africa. HTK tool that uses HMM model for to develop the system \& MFCC for feature extraction. Proposed model works on both 10 digits \& 33 alphabets collected from 60 both male \& female speakers. The Overall accuracy of the proposed model achieves $80 \%$ [7].

Therese \& Lingam, (2015) have described speaker based language independent isolated speech recognition system proposed a model in which most widely used MFCC is used for feature extraction also $\mathrm{k}$ means algorithm is used for specific feature extraction. Here this proposed system is not only used for recognizing the speech but also for language in which speech is uttered. Vocabulary contains digits from 1 to 10 of seven different languages. Which achieves $97.14 \%$ accuracy except digit three \& seven [8].

Mishra, Biswas, \& Chandra, (2010) have describes isolated Hindi digit recognition: a comparative study which works on isolated spoken Hindi digits for which proposed model has been evaluated using both HTK \& MATLAB, Also used both HMM \& MFCC for feature extraction \& database is of 0 to 9 Hindi digits from 40 speakers, recognized here. Author observed here that the HTK works better than MATLAB in both clean \& noisy environment. This achieves an overall result of $99.2 \%$ accuracy [9].

MarutiLimkara, RamaRaob \& Vidya Sagvekarc, (2012) have described isolated digit recognition using MFCC \& DTW, Which propose an approach to recognize isolated English words from 0 to 9 from different male \& female speaker. Here recognition can be done by combining other components of proposed model which are, Endpoint detection, framing, normalization, MFCC \& DTW algorithm which they used for implementation \& recognize the isolated digits. Achievement of overall accuracy rate is $90.5 \%$ [10].

Revathi \& Venkataramani, (2011) have described speaker independent continuous speech \& isolated digit recognition using VQ \& HMM, discussed both HMM \& VQ Method, they proposed a system , which uses PLP feature extraction with both combine VQ+ HMM method, Author observed here that it performs \& shows better results, using VQ with PLP they achieve $86 \%$ \& $93 \%$ accuracy with combining both VQ + HMM. PLP feature also shows results up to $99 \% \& 100 \%$ for both VQ \& combining VQ+HMM respectively [11].

Patil, Admuthe, \& Zirmite, (2014) have described isolated digit recognition using ear microphone data using MFCC, VQ \& HMM ,here proposed model implement isolated digit into three stages, which are endpoint detection \& speech segmentation, another one include $\mathrm{d}$ feature extraction which is done by MFCC, \& at last it conducted both VQ \& HMM based classifier, works on isolated English digits [12].

Trivedi, (2013) have described a survey on English digit speech recognition using HMM, which focuses on isolated English digits from 0 to 9 by implementing it with the use of the HMM Model using MFCC feature extraction technique. Here the authors have also discussed some methods \& approaches related to the speech recognition [13].

Saksamudre, (2015) have described comparative study of isolated word recognition system for Hindi language ,which focuses on comparative study on isolated Hindi language using MFCC \& KNN as pattern classification technique. When proposed model works on 10 words, it achieves overall accuracy about $89 \%$, while for 100 words, it achieves $62.50 \%$ so it is observed here that when vocabulary size increases performance will be decreased [14].

Nandyala, (2010) have described real time isolated word speech recognition system for human computer interaction ,In which proposed model works on speaker dependent system , which is implemented using MFCC feature extraction technique \& Dynamic programming algorithm. This work is used in tourism application. System obtained $88.0 \%$ accuracy [15]. Mengdi Yuet, Ling Chen, Jie Zhang, Hong Liu, has implemented speaker age recognition based on isolated words by using SVM , Here proposed model recognize speaker's age using SVM with MFCC feature extraction technique of 4507 isolated words, which achieves $72.93 \%$ accuracy. Author also compare HMM \& SVM both \& based on that it is observed that SVM is more suitable [16].

Londhe, (2014) have described hybrid HMM/ANN based isolated Hindi word recognition , proposed a model which is hybrid model for hindi word recognition. HMM model is also used for implementation with KNN classifier. Proposed model works for ten digits with 5 speakers. Author concluded here that proposed hybrid model gives efficient \& satisfactory result. Which got success in recognition accuracy up to $89.8 \%$ [17].

Tailor, Shah, Patel, \& Graduate, (2015) have described review on speech recognition system for Indian language in which author survey on different Indian languages also discussed different techniques \& expiremental results related to them. They observed some challenges related to their \& found that proper noise removal techniques improve accuracy level [18].

Dhandhania, Hansen, Kandi, \& Ramesh, (2012) have described a robust speaker independent speech recognizer for isolated Hindi digits in which proposed model works on 30 individual that represent 5 distinct age groups of 15 to 40 years for isolated 0 to 9 hindi digits using HMM Model with MFCC feature extraction that achieves $75 \%$ accuracy [19]. 
Abushariah, Gunawan, Khalifa, \& Abushariah, (2010) have described English digit speech recognition system based on HMM proposed model to implement English isolated digit from zero to nine using HMM with MATLAB. Proposed model which achieves accuracy in clean environment isolated

Karpagavalli, Rani, Deepika, \& Kokila, (2012) have described isolated tamil digit speech recognition using VQ in which proposed model is based on small vocabulary isolated speaker independent tamil digits. In which tamil digit recognizer is designed \& analyzed. Here in proposed system, codebook for each word in the vocabulary is done using Linde-Budo-Gray (LBG) vector quantization algorithm.Each digit is evaluated using word error rate \& word recognition rate. Which achieves overall $91.8 \%$ accuracy [50].

Darabkh, Khalifeh, Bathech, \& Sabah, (2013) have described Efficient DTW based speech recognition system for isolated words of Arabic language in which proposed system is used to do many tasks. Here signal is preprocessed to reduce noise effect.signal is digitized, voice activity regions are segmented

\section{PREDOMINANTLY USED TECHNIQUES IN ISOLATED SPEECH RECOGNITION}

Table 2. Techniques used in isolated speech recognition

\begin{tabular}{|c|c|c|c|}
\hline Method & Property & Advantages & Procedure for Implementation \\
\hline DTW & $\begin{array}{l}\text { Dynamic time warping is a algorithm used } \\
\text { for pattern matching.which provides time } \\
\text { registration between reference pattern \& } \\
\text { test pattern }\end{array}$ & $\begin{array}{l}\text { Used in many } \\
\text { applications like } \\
\text { speaker recognition \& } \\
\text { online signature } \\
\text { recognition }\end{array}$ & $\begin{array}{l}\text { DTW is a method that allows a computer } \\
\text { to find an optimal match between two } \\
\text { given sequences (e.g. Time series) with } \\
\text { certain restrictions. }\end{array}$ \\
\hline HMM & $\begin{array}{l}\text { Hidden Markov Model is used to study } \\
\text { hidden \& unobserved state. It constructs a } \\
\text { statistical model where each word is the } \\
\text { vocabulary \& to recognize each input word } \\
\text { as the word of the vocabulary. }\end{array}$ & $\begin{array}{l}\text { It provides a } \\
\text { framework which is } \\
\text { useful in mathematical } \\
\text { computations }\end{array}$ & Used widely in finding pairwise alignment \\
\hline LPC & $\begin{array}{l}10 \text { to } 16 \text { lower sequence coefficient, Static } \\
\text { feature extraction method }\end{array}$ & $\begin{array}{l}\text { Spectral analysis is done } \\
\text { with fixed resolution } \\
\text { along subjective } \\
\text { frequency scale }\end{array}$ & $\begin{array}{l}\text { Linear predictive coding used for feature } \\
\text { extraction at lower order }\end{array}$ \\
\hline MFCC & $\begin{array}{l}\text { Mel frequency cestrum used for find } \\
\text { features }\end{array}$ & $\begin{array}{l}\text { Provide more accurate } \\
\text { result for feature } \\
\text { extraction. }\end{array}$ & $\begin{array}{l}\text { In this method power cestrum is } \\
\text { computed by implementing fourier } \\
\text { analysis. }\end{array}$ \\
\hline VQ & $\begin{array}{l}\text { Vector Quantization is a text } \\
\text { independent recognition method which is } \\
\text { divided into two parts (i) feature training } \\
\text { (ii) feature matching where feature training } \\
\text { referred with randomly choosing feature } \\
\text { vector \& perform training using vq } \\
\text { algorithm. }\end{array}$ & $\begin{array}{l}\text { VQ has small number of } \\
\text { feature vector used as } \\
\text { specifying speaker } \\
\text { specific features. }\end{array}$ & VQ is stronger than continuous $\mathrm{hmm}$ \\
\hline
\end{tabular}

word with multi speaker 99.5 \& with speaker independent 79.5 same with Continuous Speech Recognition 92.5 , 76.67 respectively, In noisy environment isolated word with multi speaker 88 \& with speaker independent 67 same with Continuous Speech Recognition 72.5, 56.25 respectively[20]. using voice activity detection (VAD) algorithm \& features are extracted from speech signals using MFCC feature extraction technique. Proposed system added delta \& acceleration coefficients to improve recognition accuracy. Here each word's features is compared using DTW algorithm which

Das \& Parekh, (2012) have described recognition of isolated words using features based on LPC, MFCC, ZCR \& STE, with neural network classifiers in which proposed system works on isolated word corresponds to English digits from 0 to 9 which is spoken by 28 speakers that is implemented using different type of feature extraction techniques like MFCC,LPC,ZCR \& STE. This proposed system also uses ANN classifier. That achieves $85 \%$ accuracy level [52]. achieves $98.5 \%$ accuracy [51]. 


\section{ANALYSIS \& DISCUSSION}

Table 3. Comparison of isolated digit in different languages using different techniques

\begin{tabular}{|c|c|c|c|c|c|}
\hline Work & Author & Dataset & $\begin{array}{l}\text { Algorithm / Tools \& } \\
\text { Techniques }\end{array}$ & Accuracy & Language \\
\hline $\begin{array}{l}\text { Isolated } \\
\text { handwritten } \\
\text { digits }\end{array}$ & $\begin{array}{l}\text { Gattal, Abdeljalil } \\
\text { Chibani, Youcef } \\
\text { Djeddi, Chawki } \\
\text { Siddiqi, Imran [34] }\end{array}$ & $\begin{array}{l}\text { Handwritten } \\
\text { English } \\
\text { digits(0 to } 9)\end{array}$ & SVM & $\begin{array}{l}97.74 \% \& 96.72 \% \\
\text { with two proposed } \\
\text { systems respectively }\end{array}$ & English \\
\hline $\begin{array}{l}\text { Isolated } \\
\text { Digit }\end{array}$ & $\begin{array}{l}\text { Abushariah, Ahmad A M } \\
\text { Gunawan, Teddy S. } \\
\text { Khalifa, Othman O. } \\
\text { Abushariah, Mohammad } \\
\text { A M[20] }\end{array}$ & $\begin{array}{l}\text { English } \\
\text { Digits From } \\
0 \text { To } 9\end{array}$ & HMM & $\begin{array}{l}\text { Clean environment - } \\
\text { Based on Multi speaker } \\
99.5 \% \\
\text { Speaker } \\
\text { independent: } 79.5 \% \\
\text { Continuous digit: } \\
92.5 \% \\
\text { In noisy } \\
\text { environment speaker } \\
\text { independent: } 72.5 \% \text { \& } \\
56.25 \% \text { respectively }\end{array}$ & English \\
\hline $\begin{array}{l}\text { Isolated } \\
\text { digit }\end{array}$ & $\begin{array}{c}\text { Safäa ELOUAHABI } \\
\text {,Mohamed ATOUNTI, } \\
\text { Mohamed BELLOUKI [7] }\end{array}$ & $\begin{array}{l}\text { Amazigh } \\
\text { isolated digit } \\
\text { from (0 to 9) } \\
\& 33 \\
\text { alphabets }\end{array}$ & $\begin{array}{l}\text { HMM, MFCC, HTK } \\
\text { toolkit }\end{array}$ & $80 \%$ & $\begin{array}{l}\text { Amazigh } \\
\text { (spoken in } \\
\text { Africa) }\end{array}$ \\
\hline $\begin{array}{l}\text { Isolated } \\
\text { digit }\end{array}$ & Shanthi Therese S[8] & $\begin{array}{l}\quad \text { Isolated } \\
\text { digit from }(0 \\
\text { to } 9) \text { of } \\
\text { Seven } \\
\text { different } \\
\text { languages }\end{array}$ & $\begin{array}{l}\text { MFCC, K-mean } \\
\text { algorithm }\end{array}$ & $\begin{array}{l}97.14 \% \text {, except digit } 3 \\
\& 7\end{array}$ & \begin{tabular}{l}
\multicolumn{1}{c}{7} \\
different \\
languages
\end{tabular} \\
\hline $\begin{array}{l}\text { Isolated } \\
\text { digit }\end{array}$ & \begin{tabular}{l}
\multicolumn{1}{c}{ MarutiLimkara, } \\
RamaRaob \& \\
VidyaSagvekarc[10]
\end{tabular} & $\begin{array}{l}\text { English } \\
\text { isolated digit } \\
(0 \text { to } 9)\end{array}$ & DTW, MFCC & $90.50 \%$ & English \\
\hline $\begin{array}{l}\text { Isolated } \\
\text { digit }\end{array}$ & $\begin{array}{l}\text { Chapaneri, Santosh V } \\
\text { Jayaswal, Deepak J[5] }\end{array}$ & $\begin{array}{l}\text { English } \\
\text { digit from (0 } \\
\text { to } 9)\end{array}$ & $\begin{array}{l}\text { DTW,MFCC,WMFCC,I } \\
\text { FDTW }\end{array}$ & $99.16 \%$ & English \\
\hline $\begin{array}{l}\text { Isolated } \\
\text { digit }\end{array}$ & $\begin{array}{l}\text { Sabah, Reem } \\
\text { Ainon,Raja N.[36] }\end{array}$ & $\begin{array}{c}\text { Isolated } \\
\text { digit }(0 \text { to } 9)\end{array}$ & $\begin{array}{l}\text { Adaptive Neuro Fuzzy } \\
\text { Inference System } \\
\text { (ANFIS) Structure }\end{array}$ & $85.24 \%$ & Malay \\
\hline $\begin{array}{l}\text { Isolated } \\
\text { digit }\end{array}$ & Tarun Et.Al [23] & $\begin{array}{l}\text { Vocabulary } \\
\text { Of Hindi } \\
\text { Digits }\end{array}$ & $\begin{array}{l}\text { Noise Elimination, } \\
\text { LPC, HMM, VQ }\end{array}$ & $84.27 \%$ & Hindi \\
\hline $\begin{array}{l}\text { Isolated } \\
\text { digit }\end{array}$ & Bush Et.Al [26] & $\begin{array}{l}\text { Isolated } \\
\text { Digits }\end{array}$ & VQ & $96 \%$ To $97 \%$ & English \\
\hline $\begin{array}{l}\text { Isolated } \\
\text { digit }\end{array}$ & K H Davis Et.Al[21] & $\begin{array}{l}\text { Isolated } \\
\text { Telephone } \\
\text { Digits }\end{array}$ & MFCC & $97 \%$ To $99 \%$ & English \\
\hline $\begin{array}{l}\text { Isolated } \\
\text { digit }\end{array}$ & $\begin{array}{l}\text { Ike Novita } \\
\text { Dewi, } \\
\text { Fahri } \\
\text { Firdausillah } \\
\text { And }\end{array}$ & $\begin{array}{l}\text { Indonesian } \\
\text { Isolated Digit }\end{array}$ & MFCC, HMM & $50 \%$ & Indonesian \\
\hline
\end{tabular}




\begin{tabular}{|c|c|c|c|c|c|}
\hline & $\begin{array}{l}\text { Catur } \\
\text { Supriyanto[47] }\end{array}$ & & & & \\
\hline $\begin{array}{l}\text { Isolated } \\
\text { digit }\end{array}$ & $\begin{array}{l}\text { Sumit Kumar } \\
\text { Ghanty, } \\
\text { Soharab } \\
\text { Hossain } \\
\text { Shaikh And[48] }\end{array}$ & $\begin{array}{l}\text { Bengali } \\
\text { Numerals }\end{array}$ & \begin{tabular}{l}
\multicolumn{1}{c}{ MFCC, DTW WITH } \\
EUCLIDIAN \\
DISTANCE
\end{tabular} & $90 \%$ & Bengali \\
\hline $\begin{array}{l}\text { Isolated } \\
\text { digit }\end{array}$ & Karpagavalli,S & $\begin{array}{l}\text { Isolated } \\
\text { Tamil Digits }\end{array}$ & MFCC, DTW, HMM & $\begin{array}{l}\text { DTW- 87.8\% } \\
\text { HMM- 92\% }\end{array}$ & Tamil \\
\hline
\end{tabular}

The above tabular representation of table 3 shows the comparative analysis of research found in isolated digit recognition in English \& other similar languages using various techniques. In which, many attempts have been made by combining the techniques, that support to develop robust $\&$ Multilanguage isolated digit recognition. For recognizing isolated speech, two different feature extraction techniques MFCC \& LPC played pivotal role in analysing \& drawing good results. Different models are used to recognize the isolated digit, including HMM, DTW, VQ with a different toolkit namely MATLAB, HTK etc. All these techniques are compared here in table 3 among different languages. From that comparative analysis, it is observed that most of the research work in English isolated digit recognition system has been developed very far rather than other different languages.

Table 4. Comparison of isolated digit in Hindi language using different techniques

\begin{tabular}{|c|c|c|c|c|c|}
\hline Work & Author & Dataset & $\begin{array}{l}\text { Algorithm / Tools \& } \\
\text { Techniques }\end{array}$ & Accuracy & Language \\
\hline $\begin{array}{l}\text { Isolated } \\
\text { Hindi Digit }\end{array}$ & $\begin{array}{l}\text { Vedant Dhandhania, } \\
\text { Jens Kofod Hansen, } \\
\text { Shefali Jayanth } \\
\text { Kandi, And Arvind } \\
\text { Ramesh[19] }\end{array}$ & $\begin{array}{l}\text { Hindi Digit From } \\
(0 \text { To } 9)\end{array}$ & MFCC,HMM & $75 \%$ & Hindi \\
\hline $\begin{array}{l}\text { Isolated } \\
\text { Hindi Digit }\end{array}$ & $\begin{array}{l}\text { Moustafa, Akram } \\
\mathrm{A}[37]\end{array}$ & Hindi Digits (0 to 9 ) & $\begin{array}{l}\text { LPC,MFCC,VQ Also } \\
\text { Combining This Both } \\
\text { Technique }\end{array}$ & $\begin{array}{l}\mathrm{LPC}=80.6 \%, \\
\mathrm{MFCC}=96.8 \%, \\
\mathrm{LPC}+\mathrm{MFCC}+\mathrm{VQ}=9 \\
5.2 \%, \\
\mathrm{LPC}+\mathrm{MFCC}=96.1 \%\end{array}$ & Hindi \\
\hline $\begin{array}{l}\text { Isolated } \\
\text { Hindi Digit }\end{array}$ & $\begin{array}{l}\text { Mishra, A N } \\
\text { Biswas, Astik } \\
\text { Chandra, } \\
\text { Mahesh[38] }\end{array}$ & $\begin{array}{l}\text { Hindi } \\
\text { (0 to } 9 \text { ) }\end{array}$ & $\begin{array}{l}\text { MFCC,HMM,VQ,HTK,M } \\
\text { ATLAB }\end{array}$ & $99.20 \%$ & Hindi \\
\hline $\begin{array}{l}\text { Isolated } \\
\text { Hindi Digit }\end{array}$ & Tarun Et.Al [23] & $\begin{array}{l}\text { Vocabulary Of Hindi } \\
\text { Digits } \\
(0 \text { to } 9)\end{array}$ & $\begin{array}{l}\text { Noise Elimination, LPC, } \\
\text { Hmm, VQ }\end{array}$ & $84.27 \%$ & Hindi \\
\hline
\end{tabular}

Above tabular representation of table 4 discussed separately for Hindi isolated digit recognition system about the how different types of research work has been carried out to develop Hindi language. Also, their recognition rate with different combination of techniques. 
Table 5. Comparison of isolated digit in Gujarati language using different techniques

\begin{tabular}{|c|c|c|c|c|c|}
\hline Work & Author & Dataset & $\begin{array}{c}\text { Algorithm / Tools \& } \\
\text { Techniques }\end{array}$ & Accuracy & Language \\
\hline $\begin{array}{l}\text { Isolated } \\
\text { Gujarati } \\
\text { Numeral }\end{array}$ & H.B.Chauhan [3] & $\begin{array}{l}\text { Gujarati Words \& } \\
\text { Digits }\end{array}$ & MFCC \& LPC & $\begin{array}{l}\text { LPC }=85 \% \\
\text { ABOVE } \\
\text { MFCC }=95 \%\end{array}$ & Gujarati \\
\hline $\begin{array}{l}\quad \text { Isolated } \\
\text { Gujarati } \\
\text { Digits }\end{array}$ & $\begin{array}{l}\text { Purnima Pandit, } \\
\text { Shardav Bhatt[4] }\end{array}$ & Gujarati digits(0 to 9$)$ & $\begin{array}{l}\text { MFCC, DTW, } \\
\text { MATLAB }\end{array}$ & $\begin{array}{l}84 \% \\
\text { Experimental } \\
\text { result, } \\
\text { With extra care } \\
=\text { achieve } 95.56 \% \\
\text { accuracy }\end{array}$ & Gujarati \\
\hline $\begin{array}{l}\quad \text { Isolated } \\
\text { Gujarati } \\
\text { Digits }\end{array}$ & $\begin{array}{l}\text { Bhoomika Dave, D. } \\
\text { S. Pipalia [40] }\end{array}$ & $\begin{array}{l}\text { 10Guajrati digits, } 20 \\
\text { words, }\end{array}$ & MATLAB, MFCC, HMM & $\begin{array}{l}70.57 \% \text { Within } \\
10 \text { speakers } \\
\text { It increases after } \\
\text { implementation to } \\
79.14 \%\end{array}$ & Gujarati \\
\hline $\begin{array}{l}\quad \text { Isolated } \\
\text { Gujarati } \\
\text { Digits }\end{array}$ & $\begin{array}{l}\text { Patel, Bharat C } \\
\text { Desai,Apurva A [6] }\end{array}$ & $\begin{array}{l}\text { Spoken Gujarati } \\
\text { numeral from (0 to 9) }\end{array}$ & $\begin{array}{l}\text { MFCC, KNN Classifier, } \\
\text { MATLAB }\end{array}$ & $78.13 \%$ & Gujarati \\
\hline $\begin{array}{l}\quad \text { Isolated } \\
\text { Gujarati } \\
\text { Digits }\end{array}$ & $\begin{array}{l}\text { J Baheti M } \\
\text { V., Kale K } \\
\text { E Jadhav M [41] }\end{array}$ & $\begin{array}{l}\text { Isolated Gujarati } \\
\text { handwritten numeral } \\
\text { ( } 0 \text { to } 9)\end{array}$ & PCA,KNN & $\begin{array}{l}\mathrm{KNN}=90 \% \\
\mathrm{PCA}=84 \%\end{array}$ & Gujarati \\
\hline
\end{tabular}

There have been many literatures in isolated digit recognition system for the English language. Only a few attempts have been made to Gujarati language. That is discussed separately in table 5. Which represents, how different types of research work have been proposed in Gujarati language, it also shows a comparison between combinations of techniques \& their recognition rates.

Table 6. Comparison of different types of work in isolated word in different languages

\begin{tabular}{|c|c|c|c|c|c|}
\hline Work & Author & Dataset & $\begin{array}{l}\text { Algorithm / Tools \& } \\
\text { Techniques }\end{array}$ & Accuracy & Language \\
\hline $\begin{array}{l}\text { Punjabi } \\
\text { Isolated } \\
\text { Word } \\
\text { Recognition }\end{array}$ & Sharma Et.Al[31] & $\begin{array}{l}\text { Isolated } \\
\text { Punjabi Words }\end{array}$ & $\begin{array}{l}\text { Survey On All Techniques } \\
\text { Related To Isolated Word } \\
\text { Recognition }\end{array}$ & $\begin{array}{l}\text { Different } \\
\text { Accuracies As } \\
\text { Per Different } \\
\text { Techniques }\end{array}$ & Punjabi \\
\hline $\begin{array}{l}\text { Isolated } \\
\text { Word }\end{array}$ & Ishan[30] & $\begin{array}{l}\text { Hindi Isolated } \\
\text { Word }\end{array}$ & MFCC \& HMM & $97.5 \% 99 \%$ & Hindi \\
\hline $\begin{array}{l}\text { Isolated } \\
\text { Word }\end{array}$ & G V Rao[29] & Isolated Word & MFCC & $85 \%$ & Indian \\
\hline $\begin{array}{l}\text { Isolated } \\
\text { Word }\end{array}$ & $\begin{array}{l}\text { Ms.Puspa Machhar, } \\
\text { Mr.Dipak Agrawal[39] }\end{array}$ & $\begin{array}{l}\text { Gujarati } \\
\text { Tricky Words }\end{array}$ & $\begin{array}{l}\text { HMM,MFCC,KNN } \\
\text { CLASSIFIER,MATLAB }\end{array}$ & $\begin{array}{l}\text { Good Accuracy } \\
\text { Using Hmm } \\
\text { Rather Than Knn } \\
\text { Classifier }\end{array}$ & Gujarati \\
\hline $\begin{array}{l}\text { Isolated } \\
\text { Word }\end{array}$ & Dua Et.Al [24] & Isolated Word & $\begin{array}{l}\text { MFCC \& HMM WITH JAVA } \\
\& \text { HTK TOOLKIT }\end{array}$ & $\begin{array}{l}94.08 \% \text { To } \\
95.63 \%\end{array}$ & Punjabi \\
\hline $\begin{array}{l}\text { Isolated } \\
\text { Word }\end{array}$ & Kaur Et.Al[32] & Isolated Word & $\begin{array}{l}\text { Ste \& Endpoint Detection } \\
\text { Algorithm }\end{array}$ & $\begin{array}{l}\text { Accurate } \\
\text { Comparable }\end{array}$ & Punjabi \\
\hline
\end{tabular}




\begin{tabular}{|c|c|c|c|c|c|}
\hline $\begin{array}{l}\text { Isolated } \\
\text { Word }\end{array}$ & Rabinar Et.Al V[33] & $\begin{array}{l}54 \text { Isolated } \\
\text { Word }\end{array}$ & MFCC & Better Accuracy & English \\
\hline \multirow{5}{*}{$\begin{array}{l}\text { Compare } \\
\text { HMM / } \\
\text { DTW \& } \\
\text { MFCC/LPC }\end{array}$} & Rabinar Et.Al[25] & $\begin{array}{l}\text { Speaker } \\
\text { Independent } \\
\text { Isolated Words }\end{array}$ & LPC & $95 \%$ & English \\
\hline & Rupali S .Chavan [35] & Isolated word & $\begin{array}{l}\text { GMM,HMM,MFCCC,MATL } \\
\text { AB }\end{array}$ & $\begin{array}{l}\text { For Two Words- } \\
92 \% \& \text { For Third } \\
\text { Word }-88 \%\end{array}$ & English \\
\hline & L R Rabinar Et.Al [22] & Isolated word & $\begin{array}{l}\text { LPC,DTW } \\
\end{array}$ & $\begin{array}{l}\text { LPC Is Better } \\
\text { Than DTW }\end{array}$ & English \\
\hline & R Kumar [28] & $\begin{array}{l}500 \text { Punjabi } \\
\text { Isolated Word }\end{array}$ & LPC,MFCC,DTW,HMM & $\begin{array}{l}\text { HMM :92\% } \\
\text { DTW :96\% }\end{array}$ & Punjabi \\
\hline & S C .Saijjan[27] & $\begin{array}{l}\text { Isolated } \\
\text { Limited Word }\end{array}$ & LPC,MFCC,HMM,DTW & $\begin{array}{l}\text { LPC : } 91 \% \\
\text { MFCC } 94 \%\end{array}$ & English \\
\hline
\end{tabular}

Table 6 shows the comparative analysis of different types of research found especially in isolated word recognition in English \& other similar languages using various techniques.
All these techniques \& their results are compared in table 6 among English \& other different languages.

\section{Performance of Isolated Digit recognition in different languages using different techniques}

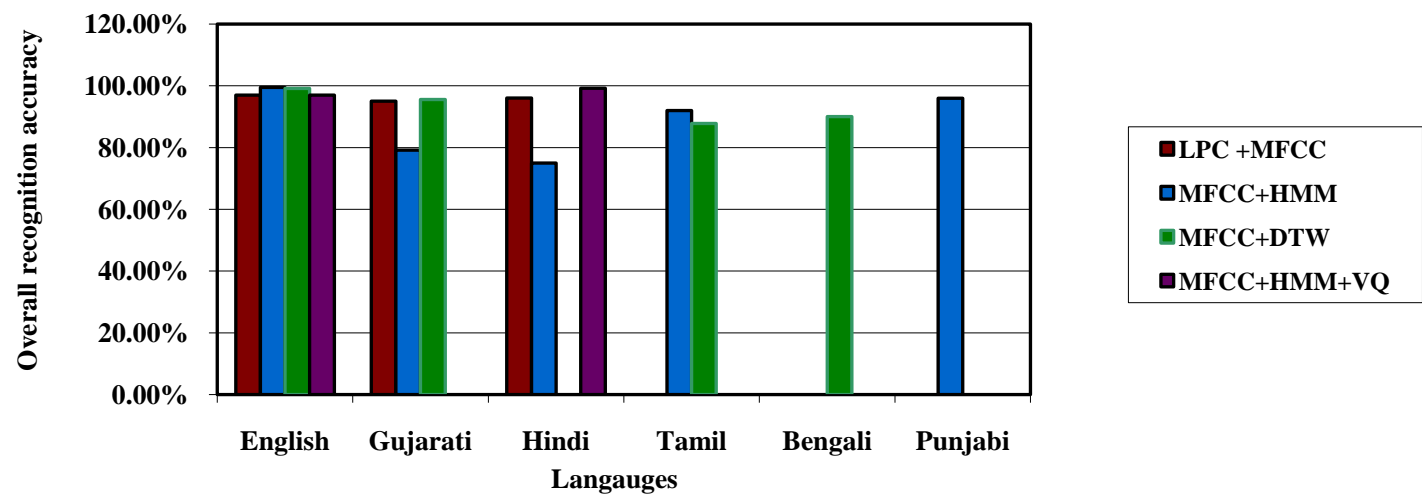

Fig. 1: Comparison of isolated digit recognition rates obtained in different languages with different techniques

The graphical representation of fig. 1 shows the overall recognition accuracy of isolated digit recognition in different languages using a combination of multiple techniques. From this figure \& comparative analysis part in this section, it's observed that researchers mostly used MFCC for feature extraction. With the combination of MFCC achieve better improvement. As it shows in this figure combination of MFCC \& HMM technique achieve higher recognition rate for each language.

\section{CONCLUSION AND FUTURE WORK}

In this survey report, comparative analysis is presented on few isolated speech recognition systems developed in different languages around the world in last few decades. Furthermore, it also includes the survey \& comparative analysis of different techniques \& their accuracy in various languages, where good results has been carried out by researchers which is presented in analysis $\&$ the discussion part in section $6 \&$ thus we found that MFCC \& HMM achieve higher recognition rate as compared to the other techniques at each language. Also, it's observed that the isolated digit recognition system is developed in English language. The amount of work in other 
similar languages has not yet reached to a critical level. So in the future, for isolated digit recognition, apart from those languages require more attention.

\section{ACKNOWLEDGMENT}

Pooja Prajapati remains thankful to Prof. Miral Patel (Department Of Information Technology, GCET) for their useful discussions \& suggestions during the preparation of this technical paper.

\section{REFERENCES}

[1] Choudhary Annu, Chauhan R, S, Gupta Gautam, "Automatic Speech Recognition System for Isolated \& Connected Words of Hindi Language By Using Hidden Markov Model Toolkit (HTK)", Association of Computer Electronics and Electrical Engineers, (ACEEE) 2013

[2] Singhal Shweta, Dubey Rajesh Kumar, "Automatic Speech Recognition For Connected Words Using DTW/HMM For English /Hindi Languages ”, IEEE 2015

[3] H. B. Chauhan, Prof. B. A. Tanawala , "Comparative Study Of MFCC \& LPC Algorithms For Gujarati Isolated Word Recognition ” , IJIRCCE , FEB-2015, VOL.3, ISSUE 2

[4] Pandit Purnima, Bhatt Shardav, "Automatic Speech Recognition Of Gujarati Digits Using Dynamic Time Warping ”, IJEIT , June-2014, VOL.3, ISSUE 12

[5] Chapaneri, Santosh V, Jayaswal, Deepak J, "Efficient Speech Recognition System For Isolated Digits", IJCSET, 2013, VOL.4, ISSUE 3, pp (228-236)

[6] Patel Bharat C, Desai Apurva A, "Recognition of Spoken Gujarati Numeral and Its Conversion into Electronic Form ”, IJERT, 2014, VOL.3, ISSUE 9

[7] Safäa ELOUAHABI, Mohamed ATOUNTI, Mohamed BELLOUKI, "Amazigh Isolated Word Speech Recognition System Using Hidden Markov Model Toolkit (HTK) ”, IEEE, 2016

[8] Therese S Shanthi, Lingam Chelpa , "Speaker Based Language Independent Isolated Speech Recognition System", IEEE, 2015

[9] Mishra A N, Biswas Astik, Chandra, Mahesh, "Isolated Hindi Digit Recognition: A Comparative Study, International Journal of Electronics and Communication Engineering (IJECE)", Jan-2010, VOL.3, ISSUE 1, pp (229-238)

[10] MarutiLimkara, RamaRaob \& Vidya Sagvekarc, "Isolated Digit Recognition Using MFCC \& DTW", IJAEEE, 2012 , VOL.1, ISSUE 1, pp (59-64)

[11] Revathi A, Venkataramani Y, “ Speaker Independent Continuous Speech \& Isolated Digit Recognition Using VQ \& Hmm ”, IEEE , 2011, pp(198-202)

[12] Patil Mahesh K, Admuthe, Prof L S , Zirmite, Prashant P , "Isolated Digit Recognition Using Ear Microphone Data Using MFCC, VQ \& HMM" , IJETT, Jun-2014 , VOL.12, ISSUE 7, pp (322-325)

[13] Vaibhavi Trivedi , "A Survey On English Digit Speech Recognition Using HMM ",VOL.2, ISSUE 3, IJSER , 2013, pp (247-253)

[14] Saksamudre, Suman K, "Comparative Study Of Isolated Word Recognition System For Hindi Language" , IJERT, JUL-2015, VOL.4, ISSUE 7 ,pp (536-540)
[15] Nandyala, Siva Prasad , “ Real Time Isolated Word Speech Recognition System For Human Computer Interaction" International Journal of Computer Applications , NOV-2010, VOL.12, ISSUE 2

[16] Mengdi Yuet, Ling Chen, Jie Zhang, Hong Liu , "Speaker Age Recognition Based On Isolated Words By Using SVM" , IEEE, 2014, pp(282-286)

[17] Londhe, Narendra D , "Hybrid HMM/ANN Based Isolated Hindi Word Recognition", IEEE,2014, ISSUE1

[18] Tailor, Jinal H, Shah, Dipti B, "Review On Speech Recognition System For Indian Language" , IJCA, Jun2015, VOL.119, ISSUE 2, pp (975-8887)

[19] Dhandhania Vedanta, Hansen Jens Kofod, Kandi Shefali Jayanth, Ramesh, Arvind, "Robust Speaker Independent Speech Recognizer For Isolated Hindi Digits ", IJCCE, Nov-2012, VOL.1, ISSUE 4, pp (483485)

[20] Abushariah, Ahmad A M, Gunawan Teddy S, Khalifa, Othman, Abushariah, Mohammad A M , "English Digit Speech Recognition System Based On HMM ", IEEE , May-2010, pp (11-13)

[21] K . H .Davis, R Biddulph \& S balashek, "Automatic Speech Recognition of Spoken Digits", J.A.S.A, 1952 ,VOL.24, ISSUE 6, pp (637-642)

[22] L R Rabiner ,A E Rosenberg \& S E Levinson , “ Considerations In Dynamic Time Warping Also For Discrete Word Recognition", IEEE , Dec -1978, Vol.1

[23] T Pruthi , S Saksena \& P K Das, "Isolated Word Recognition For Hindi Language Using VQ \& Hmm ", IIT-Madras , ICMPS

[24] M Dua R K Agrgarwal, V Kadayan \& S.Dua, " Punjabi Automatic Speech Recognition Using HTK", IJCSI, Jul2012, Vol.9

[25] L R RABINAR, M R Sambur, “ Voiced -Unvoiced Silence Detection Using LPC Distance Measure "VOL.2 , $p p(323-326)$, IEEE international conference on ICASSP

[26] M A Bush , G E Kopec \& N Lauritzen, " Segmentation In Isolated Word Recognition Using VQ" VOL-9, IEEE, 1984 , international conference on ICASSP.

[27] S C Sajjan \& C. Vijaya, "Comparison Of DTW \& HMM For Isolated Word Recognition”, IEEE, 2012, PP (466470)

[28] R Kumar , "Comparison Of HMM \& DTW For Isolated Word Recognition System For Punjabi Language", IJSC ,2010, Vol.5, Pp(88-92)

[29] Ramona Rao ,G.V \& Srichand J ,"Word Boundary Detection Using Pitch variations ", pp(813-816), International Conference On Spoken Language ICSLP , May-1996

[30] I Bhardwaj \& N D Londhe, "Hidden Markov Model Based Isolated Hindi Word Recogntion ” IEEE, 2012,

[31] A.Sharma \& A Kaur , “ Automatic Segmentation Of Punjabi Speech Into Syllable Like Units Using Group Delay A Review " , IJCSET , Jun-2013, VOL.4, pp (2229-3345)

[32] G Kaur ,P Singh \& A Kaur , “ Syllable Boundary Detection System For Punjabi Language", Vol.1, IJARC July-2013 
[33] L R Rainer \& M.R.Sambur, "AN Algorithm For Determining The Endpoints Of Isolated Utterances" , 1975,pp(297-315), The Bell System Technical Journal

[34] Gattal, Abdeljalil , Chibani, Youcef , Jedi, Chawki, Siddiqi, Imran , "Improving Isolated Digit Recognition Using a Combination of Multiple Features", IEEE, 2014

[35] Ms. Rupali S Chavan , "An Implementation of Text Dependent Speaker Independent Isolated Word Speech", IJESRT, 2013,VOL.2, ISSUE 9

[36] Sabah, Reem, Ainon Raja N , "Isolated digit speech recognition in Malay language using neuro-fuzzy approach", IEEE , 2009 , pp(336-340)

[37] Mustafa, Akram A , "Performance Evaluation of Artificial Neural Networks for Isolated Hindi Digit Recognition with LPC and MFCC" , IEEE 2015,vol.4, issue 4

[38] Mishra A N,Biswas, Astik, Chandra Mahesh, "Isolated Hindi Digits Recognition: A Comparative Study" , IJECE, 2010,Vol.3,Issue 1

[39] Ms.Puspa Machhar, Mr.Dipak Agrawal, "HMM Based Gujarati Tricky Words Recognition”, IJARIIE, 2016, Vol.2, Issue 3

[40] Bhoomika Dave, D. S. Pipalia, "An Approach To Increase Word Recognition Accuracy In Gujarati Language", International Journal Of Innovative Research In Computer And Communication Engineering, 2015, Vol.3, Issue 10

[41] J Baheti, M,V Kale K,E Jadhav M, "Comparison Of Classifiers For Gujarati Numeral Recognition”, 2011, Vol.3, Issue 3, international Journal Of Machine Intelligence

[42] M.A.Anusuya, S.K.Katti , "Speech Recognition By Machine: A Review", Ijcsis 2009 , Vol. 6, No. 3

[43] Shall Gujral , Monika Tuteja , Baljit Kaur , "Various Issues In Computerized Speech Recognition Systems " ,International Journal Of Engineering Research And General Science, June-July 2014, Volume 2, Issue 4

[44] Santosh K.Gaikwad, Bharti W.Gawali , Pravin Vannaxar " $\Delta$ Dariax On Speech Recognition IJCATM : www.ijcaonline.org Journal Of Computer 10 , Volume 10

[45] Suma Swamy1 And K.V Ramakrishnan, "An Efficient Speech Recognition System" , Computer Science \& Engineering: An International Journal (CSEIJ), August 2013, Vol. 3, No. 4

[46] Neha Chadha, R.C. Gangwar, Rajeev Bedi , "Current Challenges and Application of Speech Recognition Process using Natural Language Processing: A Survey", IJCA, Dec-2015, vol.131
[47] Ika Novita Dewi, Fahri Firdausillah, Catur Supriyanto , "Sphinx-4 Indonesian Isolated Digit Speech Recognition", Journal Of Theoretical And Applied Information Technology (JATIT), July-2013, Vol.53

[48] Sumit Kumar Ghanty , Soharab Hossain Shaikh, Nabendu Chaki, "On Recognition Of Spoken Bengali Numerals", IEEE, 2010

[49] Suman K. Saksamudre, P.P. Shrishrimal R.R. Deshmukh , " A Review On Different Approaches For Speech Recognition System”, IJCA , Apr-2015, Vol.115

[50] Karpagavalli, S, Rani, K Usha Deepika, R,Kokila, P , "Isolated Tamil Digits Speech Recognition using Vector Quantization" , International Journal of Engineering Research \& Technology (IJERT), 2012, VOL.1, ISSUE 4, $\mathrm{pp}(1-12)$

[51] Darabkh, Khalid A Khalifeh, Ala F Bathech, Baraa A Sabah, Saed W, "Efficient DTW-Based Speech Recognition System for Isolated Words of Arabic Language" ,International Journal of Computer, Electrical, Automation, Control and Information Engineering , 2013, VOL.7 , ISSUE 5, pp(586-593)

[52] Bishnu Prasad Das1, Ranjan Parekh, "Recognition of Isolated Words uses Features based on the LPC, MFCC, ZCR and STE, with Neural Network Classifiers", International Journal of Modern Engineering Research (IJMER) , May-June 2012, , Vol.2, Issue.3, pp (854858)

\section{AUTHOR PROFILE}

Pooja Prajapati completed her Bachelor of information technology from Gujarat Technological University, Gujarat, India. She is currently a research student doing her Master of information technology in G H. Patel college of Engineering \& Technology, Gujarat Technological University, Gujarat, India. Her area of interest includes speech recognition \& Artificial Intelligence.

Miral Patel completed her Bachelor of Computer Engineering from Birla Vishwakarma Mahavidyalaya, Sardar Patel University, Gujarat, India and Master of Information Technology from G H. Patel College of Engineering and Tech., Gujarat Technological University (GTU). She is currently working toward the Ph.D. degree from Changa University, Gujarat, India and have more than more than 10 Years of experience in industry and academic institutes. She has visited USA under the Gate government Project, Chicago, IL. Her Area of interest includes natural language processing, Software engineering, Project management, and Artificial intelligence. 